\title{
Stochastic Synchronization of Neutral-Type Neural Networks with Multidelays Based on $M$-Matrix
}

\author{
Wuneng Zhou, ${ }^{1,2}$ Xueqing Yang, ${ }^{1}$ Jun Yang, ${ }^{1}$ and Jun Zhou ${ }^{1}$ \\ ${ }^{1}$ College of Information Sciences and Technology, Donghua University, Shanghai 201620, China \\ ${ }^{2}$ Engineering Research Center of Digitized Textile \& Fashion Technology, Ministry of Education, Donghua University, \\ Shanghai 201620, China \\ Correspondence should be addressed to Wuneng Zhou; zhouwuneng@163.com
}

Received 24 March 2015; Revised 30 July 2015; Accepted 2 August 2015

Academic Editor: Manuel De la Sen

Copyright (C) 2015 Wuneng Zhou et al. This is an open access article distributed under the Creative Commons Attribution License, which permits unrestricted use, distribution, and reproduction in any medium, provided the original work is properly cited.

\begin{abstract}
The problem of stochastic synchronization of neutral-type neural networks with multidelays based on $M$-matrix is researched. Firstly, we designed a control law of stochastic synchronization of the neural-type and multiple time-delays neural network. Secondly, by making use of Lyapunov functional and $M$-matrix method, we obtained a criterion under which the drive and response neutral-type multiple time-delays neural networks with stochastic disturbance and Markovian switching are stochastic synchronization. The synchronization condition is expressed as linear matrix inequality which can be easily solved by MATLAB. Finally, we introduced a numerical example to illustrate the effectiveness of the method and result obtained in this paper.
\end{abstract}

\section{Introduction}

In recent years, neutral-type systems have been intensively studied due to the cause that many practical processes can be modeled as general neutral-type descriptor systems, such as computer aided design, circuit analysis, chemical process simulation, power systems, real time simulation of mechanical systems, population dynamics, and automatic control (see, e.g., [1-6] and the references therein). For example, in [1], the author studied the stability and asymptotic properties of a class of neutral-type functional differential equations based on the pattern equation method. In [2], the author investigated the asymptotic stability properties of neutraltype systems in Hilbert space.

On the one hand, time-delays as a source of instability and oscillators always appear in various aspects of neural networks. Recently, the stability of neural networks with time-delays has received lots of attention, such as $[7,8]$, and many methods, such as the linear matrix inequality (LMI) approach and $M$-matrix approach, have been adopted by the scholars; see, for example, $[9,10]$.

On the other hand, systems with Markov jump parameters, driven by continuous-time Markov chain, have been widely used to model many practical systems where they may experience abrupt changes in their structure and parameters. For example, in paper [11], a general model of an array of $\mathrm{N}$ linearly coupled delayed neural networks with Markovian jumping hybrid coupling is researched. In paper [12], the author researched the feedback control problem for a class of linear systems with Markovian jump parameters. The stabilization of stochastic delayed neural networks with Markovian switching was discussed in paper [13-16].

Meanwhile, the stability and synchronization of neutraltype systems which depend on the delays of state and state derivative have attracted a lot of attention (see [17-22] and the references therein) due to the fact that some physical systems in the real world can be described by neutral-type models. Besides the above these, according to [23, 24], $M$ matrix approach can not only design feed controller and trace all information of Markovian switching parameters but also has lower complexity than that of LMIs technology.

Inspired by the above discussions, in this paper, we are concerned with the analysis issue for the problem of stochastic synchronization of neutral-type neural networks with multidelays and Markovian switching. By using $M$ matrix approach and the stochastic analysis method, some 
synchronization criteria are obtained to ensure the stochastic synchronization for the neutral-type neural networks with multidelays. A numerical example is provided to illustrate the effectiveness of the results obtained in this paper. The main contributions of this paper are twofold: (1) Stochastic synchronization for a new class of neutral-type neural networks with multidelays and Markovian switching is considered. (2) The theory results which are obtained by $M$-method approach are more practical than that of LMIs technology.

\section{System and Problem Description and Preliminaries}

Consider $n$-dimensional multiple time-delays neutral-type neural network with Markovian switching of the form

$$
\begin{aligned}
& d[x(t)-D(r(t)) x(t-\tau)]=[-A(r(t)) x(t) \\
& +W(r(t)) \varphi(x(t)) \\
& +\sum_{k=1}^{l} W_{d k}(r(t)) \varphi\left(x\left(t-\tau_{k}(t)\right)\right) \\
& \left.+W_{d 0}(r(t)) \int_{t-\tau_{0}(t)}^{t} \varphi(x(s)) d s\right] d t
\end{aligned}
$$

where $x(t)=\left[\begin{array}{lll}x_{1}(t) & \cdots & x_{n}(t)\end{array}\right]^{T} \in \mathbb{R}^{n}$ is the state vector associated with $n$ neurons. $\varphi(x(t))=\left[\begin{array}{llll}\varphi_{1}\left(x_{1}(t)\right) & \cdots & \varphi_{n}\left(x_{n}(t)\right)\end{array}\right]^{T}$ denotes the neuron activation function; $\tau$ denotes the constant time-delay. $\tau_{k}(t), k=0,1, \ldots, l$, are time-varying delays satisfying $0<\tau_{k}(t)<\bar{\tau}$ and $\dot{\tau}_{k}(t)<\widehat{\tau}<1, k=0,1, \ldots, l$.

$\{r(t), t \geq 0\}$ is a right-continuous Markov chain on the complete probability space $\left(\Omega, \mathscr{F},\left\{\mathscr{F}_{t}\right\}_{t \geq 0}, \mathbb{P}\right)$ with a filtration $\left\{\mathscr{F}_{t}\right\}_{t \geq 0}$ satisfying the usual conditions (i.e., it is increasing and right continuous while $\mathscr{F}_{0}$ contains all $\mathbb{P}$-null sets). The Markov chain takes values in a finite state space $\mathbb{S}=$ $\{1,2, \ldots, S\}$ with generator $\Gamma=\left(\gamma_{i j}\right)_{S \times S}$ given by

$$
\begin{aligned}
\mathbb{P}\{r(t+\Delta)=j \mid r(t)=i\} & \begin{cases}\gamma_{i j} \Delta+o(\Delta) & \text { if } i \neq j \\
1+\gamma_{i i} \Delta+o(\Delta) & \text { if } i=j,\end{cases}
\end{aligned}
$$

where $\Delta>0$. Here $\gamma_{i j} \geq 0$ is the transition rate from $i$ to $j$ if $i \neq j$ while $\gamma_{i i}=-\sum_{j=1, j \neq i}^{S} \gamma_{i j}$.

$A(r(t)) \in \mathbb{R}^{n \times n}\left(A_{i}, r(t)=i\right.$, for short $)$ is a positive diagonal matrix with its diagonal elements $a_{j}, j=1,2, \ldots, n$. $W(r(t)) \in \mathbb{R}^{n \times n}$ and $W_{d k}(r(t)) \in \mathbb{R}^{n \times n}(k=0,1, \ldots, l)$ are the connection weight matrix and the time-delay connection weight matrix, respectively. $D(r(t)) \in \mathbb{R}^{n \times n}$ is external input matrix.
For system (1) (called the drive system), the response system is of the following form:

$$
\begin{aligned}
& d[y(t)-D(r(t)) y(t-\tau)]=[-A(r(t)) y(t) \\
& +W(r(t)) \varphi(y(t)) \\
& +\sum_{k=1}^{l} W_{d k}(r(t)) \varphi\left(y\left(t-\tau_{k}(t)\right)\right) \\
& \left.+W_{d 0}(r(t)) \int_{t-\tau_{0}(t)}^{t} \varphi(y(s)) d s+U(r(t))\right] d t \\
& +g\left(t, r(t), e(t), e_{\tau_{0}}(t), e_{\tau_{1}}(t), \ldots, e_{\tau_{l}}(t)\right) d \omega(t)
\end{aligned}
$$

where $y(t)=\left[\begin{array}{lll}y_{1}(t) & \cdots & y_{n}(t)\end{array}\right]^{T} \in \mathbb{R}^{n}$ is the state vector of the drive system, $U(r(t)) \in \mathbb{R}^{n}$ is the control input vector, $e(t)=y(t)-x(t), e_{\tau_{k}}(t)=y\left(t-\tau_{k}(t)\right)-x\left(t-\tau_{k}(t)\right), k=$ $0,1, \ldots, l$, and $g$ is the noise intensity function satisfying $g$ : $\mathbb{R} \times \mathbb{S} \times R^{n} \times \cdots \times \mathbb{R}^{n} \rightarrow \mathbb{R}^{n \times m}$.

$\omega(t)=\left[\omega_{1}(t), \omega_{2}(t), \ldots, \omega_{m}(t)\right]^{T}$ is $m$-dimensional $\mathscr{F}_{t^{-}}$ adapted Brownian motion. It is assumed that $\{r(t)\}$ and $\omega(t)$ in system (3) are independent.

For drive system (1) and response system (3), we can obtain the error system as follows:

$$
\begin{aligned}
& d[e(t)-D(r(t)) e(t-\tau)]=[-A(r(t)) e(t) \\
& \quad+W(r(t)) \psi(t)+\sum_{k=1}^{l} W_{d k}(r(t)) \psi_{\tau_{k}}(t) \\
& \left.\quad+W_{d 0}(r(t)) \int_{t-\tau_{0}(t)}^{t} \psi(s) d s+U(r(t))\right] d t \\
& \quad+g\left(t, r(t), e(t), e_{\tau_{0}}(t), e_{\tau_{1}}(t), \ldots, e_{\tau_{l}}(t)\right) d \omega(t),
\end{aligned}
$$

where $\psi(t)=\varphi(y(t))-\varphi(x(t))$ and $\psi_{\tau_{k}}(t)=\varphi\left(y\left(t-\tau_{k}(t)\right)\right)-$ $\varphi\left(x\left(t-\tau_{k}(t)\right)\right)$.

The initial data is given by $\{e(\theta):-\widetilde{\tau} \leq \theta \leq 0\}=\xi(\theta) \epsilon$ $\left.L_{\mathscr{F}_{0}}^{2}\left([-\tilde{\tau}, 0] ; \mathbb{R}^{n}\right]\right), r(0)=r_{0}, \xi(0)=0$, where $\tilde{\tau}=\max \{\tau, \bar{\tau}\}$. tions.

For error system (4), we impose the following assump-

Assumption 1. Each function $\varphi_{j}: \mathbb{R} \rightarrow \mathbb{R}$ is nondecreasing and there exists a positive constant $\Phi$ such that

$$
\left|\varphi_{j}(u)-\varphi_{j}(v)\right| \leq \Phi|u-v|
$$

$$
\forall u, v \in \mathbb{R}, j=1,2, \ldots, n .
$$

Assumption 2. $\forall i \in \mathbb{S}$, there exist some positive constants $G$ and $G_{i}, i=0,1, \ldots, l$, such that

$$
\operatorname{trace}\left(g^{T}(\cdot) g(\cdot)\right) \leq G|e(t)|^{2}+\sum_{k=0}^{l} G_{k}\left|e_{\tau_{k}}(t)\right|^{2},
$$

and $g(t, i, 0, \ldots, 0)=0$. 
Assumption 3. For the external input matrix $D_{i}(i \in \mathbb{S})$, there exists positive constant $\kappa_{i} \in(0,1)$, such that

$$
\rho\left(D_{i}\right)=\kappa_{i} \leq \kappa \in(0,1),
$$

where $\kappa=\max _{i \in \mathbb{S}} \kappa_{i}$ and $\rho\left(D_{i}\right)$ is the spectral radius of matrix $D_{i}$.

We now begin with the following concept of stochastic synchronization.

Definition 4. Neutral-type response neural networks (3) are said to be stochastic synchronized with drive neural network (1) if, for any $\left.\xi(t) \in L_{\mathscr{F}_{0}}^{2}\left([-\widetilde{\tau}, 0] ; \mathbb{R}^{n}\right]\right)$ and $r_{0} \in \mathbb{S}$,

$$
\int_{0}^{\infty} \mathbb{E}\left|x\left(t ; i_{0}, \xi(t)\right)\right|^{2}<\infty,
$$

where $x\left(t ; i_{0}, \xi(t)\right)$ is the solution of system (4) for the initial condition $\xi(t)$. follows.

Now, we describe the problem to solve in this paper as

Target Description. For neutral-type and multiple time-delays neural networks (1) and (3) with stochastic disturbance and Markovian switching, by using Lyapunov functional, general Itô's formula, and $M$-matrix method, this paper will design a control law and obtain some criteria of stochastic synchronization. result.

The following lemmas are useful for obtaining the main

Lemma 5 (see [25]). Let $x, y \in \mathbb{R}^{n}$; then the inequality $x^{T} y+$ $y^{T} x \leq \epsilon x^{T} x+\epsilon^{-1} y^{T} y$ holds for any $\epsilon>0$.

Lemma 6 (see [26]). For any positive definite matrix $M \in$ $\mathfrak{R}^{n \times n}$, a scalar $\gamma>0$, vector function $\omega:[0, \gamma] \rightarrow \mathfrak{R}^{n}$ such that the integration concerned is well defined; then

$$
\begin{aligned}
& \left(\int_{0}^{\gamma} \omega(s) d s\right)^{T} M\left(\int_{0}^{\gamma} \omega(s) d s\right) \\
& \quad \leq \gamma \int_{0}^{\gamma} \omega^{T}(s) M \omega(s) d s .
\end{aligned}
$$

Lemma 7 (see [27]). If $M=\left(m_{i j}\right)_{n \times n} \in \mathbb{R}^{n \times n}$ with $m_{i j}<$ $0(i \neq j)$, then the following statements are equivalent:

(i) $M$ is a nonsingular $M$-matrix.

(ii) Every real eigenvalue of $M$ is positive.

(iii) $M$ is positive stable. That is, $M^{-1}$ exists and $M^{-1}>$ 0 (i.e., $M^{-1} \geq 0$ and at least one element of $M^{-1}$ is positive).

\section{Main Results}

We are now in a position to derive a condition under which neutral-type multiple time-delay neural networks (3) with stochastic disturbance and Markovian switching are stochastic synchronized with drive system (1). We have the following result.
Theorem 8. Let Assumptions 1, 2, and 3 hold. Assume that $M:=-\operatorname{diag}\{\underbrace{\eta, \eta, \ldots, \eta}\}-\Gamma$ is a nonsingular $M$-matrix, where

$$
\eta=-2 \varsigma+\beta^{2}+\beta_{d}^{2}+\sum_{k=1}^{l} \beta_{d k}^{2},
$$

with c being a nonnegative real number, and $\beta=\max _{i \in \mathbb{S}} \rho\left(W_{i}\right)$, $\beta_{d}=\max _{i \in \mathbb{S}} \rho\left(W_{d i}\right)$, and $\beta_{d k}=\max _{i \in \mathbb{S}} \rho\left(W_{d k i}\right)$.

Let $m>0, \vec{m}=[\underbrace{m, m, \ldots, m}_{S}]^{T}$ and $\left[q_{1}, q_{2}, \ldots, q_{S}\right]^{T}:=$ $M^{-1} \vec{m}$.

Assume also that

$$
\begin{aligned}
a & =2 \underline{\alpha} q-\bar{\alpha}^{2} q-\Phi^{2} q-G_{0} q-Q_{\tau}-\sum_{k=0}^{l} Q_{k}-\bar{\tau}^{2} \Phi^{2} \\
& >0
\end{aligned}
$$

where $\underline{\alpha}=\min _{i \in \mathbb{S}} \min _{1 \leq j \leq n} a_{j}^{i}, \bar{\alpha}=\max _{i \in \mathbb{S}} \max _{1 \leq j \leq n} a_{j}^{i}, q=$ $\min _{i \in \mathbb{S}} q_{i}, Q_{\tau}=q \kappa^{2}$, and $Q_{k}=q\left(\Phi^{2}+G_{k}\right) /(1-\widehat{\tau})$.

We choose the feedback control $U_{i}$ with the update law as

$$
U_{i}=\left(\operatorname{diag}\left\{k_{1}, k_{2}, \ldots, k_{n}\right\}-\varsigma I\right)\left(e(t)-D_{i} e_{\tau}(t)\right)
$$

with

$$
\dot{k}_{j}=-\alpha_{j} q\left(e_{j}-D_{i}\left(e_{\tau}\right)_{j}\right)^{2},
$$

where $\alpha_{j}>0(j=1,2, \ldots, n)$ are arbitrary constants.

Then neutral-type multiple time-delays neural networks (3) with stochastic disturbance and Markovian switching can be stochastic synchronized with drive system (1).

Proof. Fix any $\left(i_{0}, \xi(t)\right) \in \mathbb{S} \times \mathbb{R}^{n}$ write $e\left(t ; i_{0}, \xi(t)\right)=e(t)$ for simplicity. For neutral-type multiple time-delays neural networks (4), consider the following Lyapunov functional $V \in \mathbb{C}^{2,1}\left(\mathbb{R}_{+} \times \mathbb{S} \times \mathbb{R}^{n} ; \mathbb{R}_{+}\right):$

$$
\begin{aligned}
V(t, i, e(t))= & q|e(t)|^{2}+\sum_{j=1}^{n} \frac{1}{\alpha_{j}} k_{j}^{2} \\
& +\sum_{k=0}^{l} \int_{t-\tau_{k}(t)}^{t} e^{T}(s) Q_{k} e(s) d s \\
& +\int_{t-\tau}^{t} e^{T}(s) Q_{\tau} e(s) d s \\
& +\bar{\tau} \int_{-\tau_{0}(t)}^{0} \int_{t+\alpha}^{t} \psi^{T}(s) \psi(s) d s d \alpha .
\end{aligned}
$$

Computing $\mathscr{L} V$ along neural networks (4), we can obtain

$$
\begin{aligned}
\mathscr{L} V(t, i, e(t)) \\
=V_{t}\left(t, i, e-D_{i} e_{\tau}\right)+V_{e}\left(t, i, e-D_{i} e_{\tau}\right) f\left(t, i, e, e_{\tau}\right) \\
\quad+\frac{1}{2} \operatorname{trace}\left[g^{T}(\cdot) V_{e e}\left(t, i, e-D_{i} e_{\tau}\right) g(\cdot)\right] \\
\quad+\sum_{k=1}^{S} \gamma_{i k} V\left(t, k, e-D_{i} e_{\tau}\right),
\end{aligned}
$$


where $f\left(t, i, e, e_{\tau}\right)=-A(r(t)) e(t)+W(r(t)) \psi(t)+$ $\sum_{k=1}^{l} W_{d k}(r(t)) \psi_{\tau_{k}}(t)+W_{d 0}(r(t)) \int_{t-\tau_{0}(t)}^{t} \psi(s) d s+U(r(t))$. Now,

$$
\begin{aligned}
& V_{e}\left(t, i, e-D_{i} e_{\tau}\right)=2 q\left(e-D_{i} e_{\tau}\right)^{T}, \\
& V_{e e}\left(t, i, e-D_{i} e_{\tau}\right)=2 q, \\
& V_{t}\left(t, i, e-D_{i} e_{\tau}\right) \\
& =\sum_{j=1}^{n} \frac{2}{\alpha_{j}} k_{j} \dot{k}_{j}+\sum_{k=1}^{l} e^{T}(t) Q_{k} e(t) \\
& \quad-\sum_{k=1}^{l}\left(1-\dot{\tau}_{k}(t)\right) e^{T}\left(t-\tau_{k}(t)\right) Q_{k} e\left(t-\tau_{k}(t)\right) \\
& \quad+e^{T}(t) Q_{\tau} e(t)-e^{T}(t-\tau) Q_{\tau} e(t-\tau) \\
& \quad+\bar{\tau} \tau_{0}(t) \psi^{T}(t) \psi(t)-\bar{\tau} \int_{t-\tau_{0}(t)}^{t} \psi^{T}(s) \psi(s) d s \\
& \quad-2 q \sum_{j=1}^{n} k_{j}\left(x_{j}-D_{i}\left(x_{\tau}\right)_{j}\right)^{2}+\sum_{k=1}^{l} e^{T}(t) Q_{k} e(t) \\
& \quad-\sum_{k=1}^{l}(1-\widehat{\tau}) e^{T}\left(t-\tau_{k}(t)\right) Q_{k} e\left(t-\tau_{k}(t)\right) \\
& +\quad e^{T}(t) Q_{\tau} e(t)-e^{T}(t-\tau) Q_{\tau} e(t-\tau) \\
& +\bar{\tau}^{2} \Phi^{2} e^{T}(t) e(t)-\bar{\tau} \int_{t-\tau_{0}(t)}^{t} \psi^{T}(s) \psi(s) d s .
\end{aligned}
$$

From Lemma 5, we get

$$
\begin{aligned}
-2 q\left(e-D_{i} e_{\tau}\right)^{T} A_{i} e \leq & -2 \min \rho\left(A_{i}\right) q\left(e^{T} e\right) \\
& +2 q e_{\tau}^{T} D_{i}^{T} A_{i} e \\
\leq & -2 \underline{\alpha} q|e|^{2} \\
& +q\left(e^{T} A_{i}^{T} A_{i} e+e_{\tau}^{T} D_{i}^{T} D_{i} e_{\tau}\right) \\
\leq & -2 \underline{\alpha} q|e|^{2}+q \bar{\alpha}^{2}|e|^{2}+q \kappa^{2}\left|e_{\tau}\right|^{2} \\
= & q\left(-2 \underline{\alpha}+\bar{\alpha}^{2}\right)|e|^{2}+q \kappa^{2}\left|e_{\tau}\right|^{2} .
\end{aligned}
$$

From Assumption 1 and Lemma 5, it can be computed that

$$
\begin{aligned}
2 q & \left(e-D_{i} e_{\tau}\right)^{T} W_{i} \psi(t) \\
& \leq q\left(\left(e-D_{i} e_{\tau}\right)^{T} W_{i} W_{i}^{T}\left(e-D_{i} e_{\tau}\right)+\psi^{T}(t) \psi(t)\right) \\
& \leq q\left(\max _{i \in \mathbb{S}} \rho\left(W_{i}\right)\right)^{2}\left|e-D_{i} e_{\tau}\right|^{2}+q \Phi^{2}|e|^{2} \\
& =q \Phi^{2}|e|^{2}+q \beta^{2}\left|e-D_{i} e_{\tau}\right|^{2} .
\end{aligned}
$$

Similarly,

$$
\begin{aligned}
& 2 q\left(e-D_{i} e_{\tau}\right)^{T} \sum_{k=1}^{l} W_{d k i} \psi\left(t-\tau_{k}(t)\right)=q \sum_{k=1}^{l} 2(e \\
& \left.-D_{i} e_{\tau}\right)^{T} W_{d k i} \psi\left(t-\tau_{k}(t)\right) \\
& \leq q \sum_{k=1}^{l}\left(\left(e-D_{i} e_{\tau}\right)^{T} W_{d k i} W_{d k i}^{T}\left(e-D_{i} e_{\tau}\right)\right. \\
& \left.+\psi^{T}\left(t-\tau_{k}(t)\right) \psi\left(t-\tau_{k}(t)\right)\right) \\
& \leq q \sum_{k=1}^{l}\left(\Phi^{2}\left|e\left(t-\tau_{k}(t)\right)\right|^{2}+\beta_{d k}^{2}\left|e-D_{i} e_{\tau}\right|^{2}\right) .
\end{aligned}
$$

From Lemmas 5 and 6, we have

$$
\begin{aligned}
& 2 q\left(e-D_{i} e_{\tau}\right)^{T} W_{d i} \int_{t-\tau_{0}(t)}^{t} \psi(s) d s \\
& \quad \leq q\left(W_{d i} W_{d i}^{T}\left|e-D_{i} e_{\tau}\right|^{2}\right. \\
& \left.\quad+\left(\int_{t-\tau_{0}(t)}^{t} \psi(s) d s\right)^{T} \int_{t-\tau_{0}(t)}^{t} \psi(s) d s\right) \\
& \quad \leq q\left(\beta_{d}^{2}\left|e-D_{i} e_{\tau}\right|^{2}+\bar{\tau} \int_{t-\tau_{0}(t)}^{t} \psi^{T}(s) \psi(s) d s\right) .
\end{aligned}
$$

From control law (12), one can obtain

$$
\begin{aligned}
& 2 q\left(e-D_{i} e_{\tau}\right)^{T} U_{i}=2 q\left(e-D_{i} e_{\tau}\right)^{T} \\
& \cdot \operatorname{diag}\left\{k_{1}-\varsigma, k_{2}-\varsigma, \ldots, k_{n}-\varsigma\right\}\left(e-D_{i} e_{\tau}\right) \\
& =2 q \sum_{j=1}^{n} k_{j}\left(x_{j}-D_{i}\left(x_{\tau}\right)_{j}\right)^{2}-2 q \varsigma\left|e-D_{i} e_{\tau}\right|^{2} .
\end{aligned}
$$

From Assumption 2, we have

$$
\begin{aligned}
& \left(\frac{1}{2}\right) \operatorname{trace}\left(g^{T} g\right)=q \operatorname{trace}\left(g^{T} g\right) \\
& \quad \leq q\left(G|e|^{2}+\sum_{k=0}^{l} G_{k}\left|e\left(t-\tau_{k}(t)\right)\right|^{2}\right) .
\end{aligned}
$$

Finally,

$$
\sum_{k=1}^{\mathcal{\delta}} \gamma_{i k} V\left(t, k, e-D_{i} e_{\tau}\right)=\sum_{k=1}^{\mathcal{\delta}} \gamma_{i k} q_{k}\left|e-D_{i} e_{\tau}\right|^{2}
$$


Substituting (17)-(24) into (15), we obtain that

$$
\mathscr{L} V \leq-a|e|^{2}-m\left|e-D_{i} e_{\tau}\right|^{2}<-a|e|^{2},
$$

where $m=-\left[\eta q+\sum_{k=1}^{S} \gamma_{i k} q_{k}\right]$ by $\left(q_{1}, q_{2}, \ldots, q_{S}\right)^{T}=M^{-1} \vec{m}$.

Integrating and taking the mathematical expectation on both sides of (25), one can get that

$$
\begin{aligned}
\int_{0}^{\infty} \mathbb{E}\left|x\left(t ; i_{0}, \xi(t)\right)\right|^{2} & <\left(\frac{1}{a}\right) \mathbb{E} V(0, r(0), e(0)) \\
& <\infty .
\end{aligned}
$$

Therefore, it can be concluded from Definition 4 that response system (3) synchronizes stochastically drive system (1). This completes the proof.

Remark 9. In Theorem 8, we have assumed that $M:=$ $-\operatorname{diag}\{\underbrace{\eta, \eta, \ldots, \eta}_{S}\}-\Gamma$ is a nonsingular $M$-matrix. For given Markovian rate generator $\Gamma$, networks parameters $W_{i}$ and $W_{d k i}, k=0,1, \ldots, l$, according to $M$-matrix properties (see Lemma 7), we can obtain parameter $\varsigma$. Thus, we can design feedback control update law (12). This technique is different from those, such as linear matrix inequality method [28]. From the process of proving Theorem 8 , if $M$-matrix approach is not used, and LMIs technology is only adopted to solve stochastic synchronization of addressed system (1), (25) cannot be produced. In order to make $\int_{0}^{\infty} \mathbb{E}\left|x\left(t ; i_{0}, \xi(t)\right)\right|^{2}<$ $(1 / a)<\infty$, the theory result in Theorem 8 will become more complex.

Remark 10. From the analysis of Remark 9, we can also obtain that if feedback control update law (12) has been designed, because $M:=-\operatorname{diag}\{\underbrace{\eta, \eta, \ldots, \eta}_{S}\}-\Gamma$, using $M$-matrix method, Markovian rate generator $\Gamma$ can be checked. This also reflects that all information of Markovian switching parameters is traced.

When the multidelays turn to single delay and the neutral term disappears in the neural networks, respectively, we have the following two special cases of system (4).

Special Case 1 (single delay). Consider

$$
\begin{aligned}
& d[e(t)-D(r(t)) e(t-\tau)]=[-A(r(t)) e(t) \\
& \quad+W(r(t)) \psi(t)+W_{d 1}(r(t)) \psi_{\tau_{1}}(t) \\
& \left.\quad+W_{d 0}(r(t)) \int_{t-\tau_{0}(t)}^{t} \psi(s) d s+U(r(t))\right] d t \\
& \quad+g\left(t, r(t), e(t), e_{\tau_{0}}(t), e_{\tau_{1}}(t), \ldots, e_{\tau_{l}}(t)\right) d \omega(t) .
\end{aligned}
$$

Special Case 2 (without neutral term). Consider

$$
\begin{aligned}
& d e(t)=[-A(r(t)) e(t)+W(r(t)) \psi(t) \\
& \quad+\sum_{k=1}^{l} W_{d k}(r(t)) \psi_{\tau_{k}}(t)+W_{d 0}(r(t)) \int_{t-\tau_{0}(t)}^{t} \psi(s) d s \\
& +U(r(t))] d t \\
& \quad+g\left(t, r(t), e(t), e_{\tau_{0}}(t), e_{\tau_{1}}(t), \ldots, e_{\tau_{l}}(t)\right) d \omega(t) .
\end{aligned}
$$

Accordingly we can derive the two corollaries of Theorem 8 below.

Corollary 11. Let Assumptions 1, 2, and 3 hold. Assume that $M:=-\operatorname{diag}\{\underbrace{\eta, \eta, \ldots, \eta}_{S}\}-\Gamma$ is a nonsingular $M$-matrix, where

$$
\eta=-2 \varsigma+\beta^{2}+\beta_{d}^{2}+\beta_{d 1}^{2}
$$

with $\varsigma$ being a nonnegative real number, and $\beta=\max _{i \in \mathbb{S}} \rho\left(W_{i}\right)$, $\beta_{d}=\max _{i \in \mathbb{S}} \rho\left(W_{d i}\right)$, and $\beta_{d 1}=\max _{i \in \mathbb{S}} \rho\left(W_{d 1 i}\right)$.

Let $m>0, \vec{m}=[\underbrace{m, m, \ldots, m}_{S}]^{T}$ and $\left[q_{1}, q_{2}, \ldots, q_{S}\right]^{T}:=$ $M^{-1} \vec{m}$.

Assume also that

$$
\begin{aligned}
& a=2 \underline{\alpha} q-\bar{\alpha}^{2} q-\Phi^{2} q-G_{0} q-Q_{\tau}-\sum_{k=0}^{1} Q_{k}-\bar{\tau}^{2} \Phi^{2} \\
& \quad>0
\end{aligned}
$$

where $\underline{\alpha}=\min _{i \in \mathbb{S}} \min _{1 \leq j \leq n} a_{j}^{i}, \bar{\alpha}=\max _{i \in \mathbb{S}} \max _{1 \leq j \leq n} a_{j}^{i}, q=$ $\min _{i \in \mathbb{S}} q_{i}, Q_{\tau}=q \kappa^{2}$, and $Q_{k}=q\left(\Phi^{2}+G_{k}\right) /(1-\hat{\tau})$.

We choose the feedback control $U_{i}$ with the update law as

$$
U_{i}=\left(\operatorname{diag}\left\{k_{1}, k_{2}, \ldots, k_{n}\right\}-\varsigma I\right)\left(e(t)-D_{i} e_{\tau}(t)\right)
$$

with

$$
\dot{k}_{j}=-\alpha_{j} q\left(e_{j}-D_{i}\left(e_{\tau}\right)_{j}\right)^{2}
$$

where $\alpha_{j}>0(j=1,2, \ldots, n)$ are arbitrary constants.

Then the response system can synchronize with the drive system of neutral-type.

Corollary 12. Let Assumptions 1, 2, and 3 hold. Assume that $M:=-\operatorname{diag}\{\underbrace{\eta, \eta, \ldots, \eta}_{S}\}-\Gamma$ is a nonsingular $M$-matrix, where

$$
\eta=-2 \varsigma+\beta^{2}+\beta_{d}^{2}+\sum_{k=1}^{l} \beta_{d k}^{2}
$$


with $\varsigma$ being a nonnegative real number, and $\beta=\max _{i \in \mathbb{S}} \rho\left(W_{i}\right)$, $\beta_{d}=\max _{i \in \mathbb{S}} \rho\left(W_{d i}\right)$, and $\beta_{d k}=\max _{i \in \mathbb{S}} \rho\left(W_{d k i}\right)$.

Let $m>0, \vec{m}=[\underbrace{m, m, \ldots, m}_{S}]^{T}$ and $\left[q_{1}, q_{2}, \ldots, q_{S}\right]^{T}:=$ $M^{-1} \vec{m}$.

Assume also that

$$
a=2 \underline{\alpha} q-\bar{\alpha}^{2} q-\Phi^{2} q-G_{0} q-\sum_{k=0}^{l} Q_{k}-\bar{\tau}^{2} \Phi^{2}>0,
$$

where $\underline{\alpha}=\min _{i \in \mathbb{S}} \min _{1 \leq j \leq n} a_{j}^{i}, \bar{\alpha}=\max _{i \in \mathbb{S}} \max _{1 \leq j \leq n} a_{j}^{i}, q=$ $\min _{i \in \mathbb{S}} q_{i}$, and $Q_{k}=q\left(\Phi^{2}+G_{k}\right) /(1-\widehat{\tau})$.

We choose the feedback control $U_{i}$ with the update law as

$$
U_{i}=\left(\operatorname{diag}\left\{k_{1}, k_{2}, \ldots, k_{n}\right\}-\varsigma I\right) e(t)
$$

with

$$
\dot{k}_{j}=-\alpha_{j} q e_{j}^{2}
$$

where $\alpha_{j}>0(j=1,2, \ldots, n)$ are arbitrary constants.

Then the response system can synchronize with the drive system of multiple time-delays.

\section{Numerical Simulation}

One example is presented here in order to show the usefulness of our results. Our aim is to examine the stochastic synchronization for the given neutral-type multiple timedelays neural networks with stochastic noise and Markovian switching.

Consider the error system of two-neuron delayed neural network (4) with 2-state Markovian switching and onedimensional noise $B(t)$. We set the constant time-delay $\tau=1$ and $\tau_{0}(t)=1, \tau_{1}(t)=0.8 \sin (t), \tau_{2}(t)=0.8 \cos (t)$, which means $\bar{\tau}=1$ and $\widehat{\tau}=0.8$. by

The transition rate matrix of Markovian switching is given

$$
\begin{aligned}
\Gamma & =\left[\begin{array}{cc}
-4 & 4 \\
3 & -3
\end{array}\right], \\
\varphi(\cdot) & =\frac{\tanh (\cdot)}{4}, \\
g(\cdot) & =\frac{e(t)}{2} .
\end{aligned}
$$

Then we choose $\Phi=0.25, G=0.25$, and $G_{k}=0$ so that Assumptions 1 and 2 can be satisfied.
The other parameters are given as follows:

$$
D_{1}=\left[\begin{array}{cc}
0.2 & 0 \\
0 & 0.1
\end{array}\right] \text {, }
$$

$$
\begin{aligned}
A_{1} & =\left[\begin{array}{cc}
0.9 & 0 \\
0 & 1
\end{array}\right], \\
W_{1} & =\left[\begin{array}{cc}
0.02 & -0.03 \\
0.05 & 0.04
\end{array}\right],
\end{aligned}
$$

$W_{d 01}=\left[\begin{array}{cc}0.04 & 0.03 \\ -0.03 & 0.02\end{array}\right]$,

$W_{d 11}=\left[\begin{array}{ll}0.05 & -0.02 \\ 0.03 & -0.05\end{array}\right]$,

$W_{d 21}=\left[\begin{array}{cc}0.04 & -0.02 \\ 0.01 & 0.02\end{array}\right]$,

$$
D_{2}=\left[\begin{array}{cc}
0.1 & 0 \\
0 & 0.05
\end{array}\right] \text {, }
$$$$
A_{2}=\left[\begin{array}{cc}
1 & 0 \\
0 & 0.9
\end{array}\right] \text {, }
$$

$$
W_{2}=\left[\begin{array}{cc}
-0.02 & 0.01 \\
0.02 & -0.05
\end{array}\right] \text {, }
$$

$W_{d 02}=\left[\begin{array}{ll}0.03 & -0.01 \\ 0.02 & -0.04\end{array}\right]$,

$W_{d 12}=\left[\begin{array}{ll}0.03 & -0.06 \\ 0.02 & -0.01\end{array}\right]$,

$W_{d 22}=\left[\begin{array}{cc}0.03 & 0.01 \\ -0.04 & 0.02\end{array}\right]$.

Given $\varsigma=0.5, m=1$, we compute

$$
\begin{aligned}
\eta & =-0.9923, \\
M & =\left[\begin{array}{cc}
1.4923 & -0.5 \\
-0.8 & 1.7923
\end{array}\right] .
\end{aligned}
$$

It can be easily verified that $M$ is $M$-matrix. We then derived $q=1.0078$ and $\alpha=0.0509>0$. Hence it follows from Theorem 8 that drive system (1) and response system (3) are stochastic synchronization. Figures 1-4 show the 2state Markov chain, the state trajectory of the drive system and response system, the state trajectory and evolution of the error system, and the update law of the control gain matrix, respectively. We can see from Figure 3 that the system state tends to zero with the increase of $t$, which verifies the synchronization of the drive system and response system. 


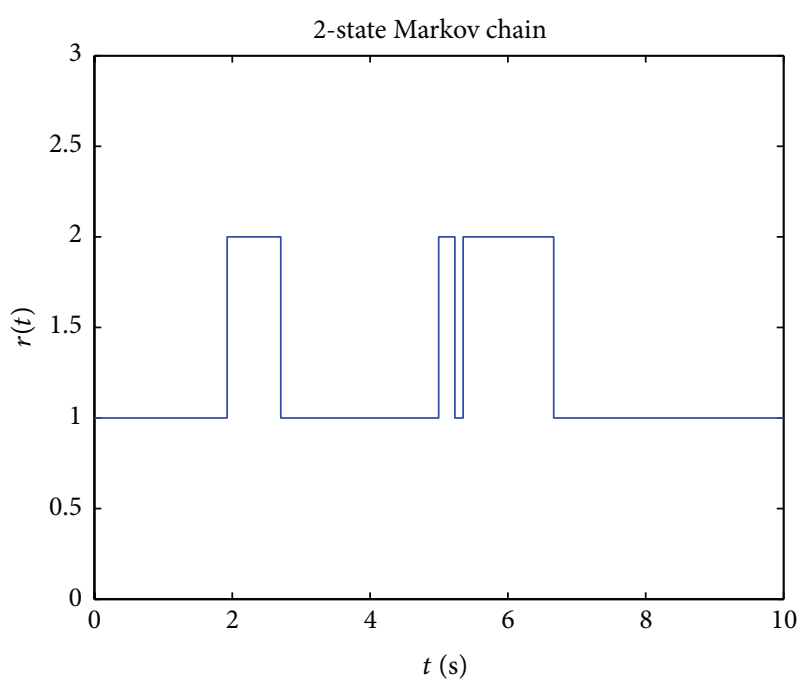

FIGURE 1: 2-state Markov chain.
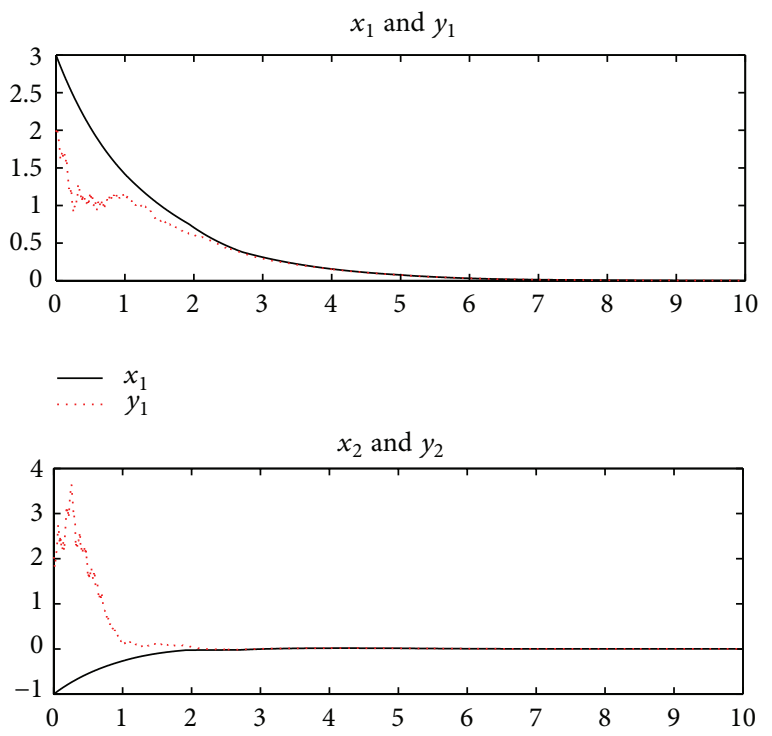

$\begin{array}{ll} & x_{2} \\ \cdots \cdots & y_{2}\end{array}$

FIGURE 2: State trajectory of two systems.

Remark 13. For Markovian switching with known transition rate, we can choose reasonable parameter $\eta$ to realize $M:=$ $-\operatorname{diag}\{\underbrace{\eta, \eta, \ldots, \eta}_{S}\}-\Gamma$ to become $M$-matrix. In simulation results, choosing $\varsigma=0.5$, it is easily observed that $M$ is $M$ matrix. Furthermore, we can get $q$ and $\alpha$. From the above result, we can see that the analysis of $M$-matrix approach in Remarks 9 and 10 is reasonable.

\section{Conclusion}

In this paper, we have dealt with the problem of stochastic synchronization of neutral-type neural networks with multidelays and Markovian switching. By using $M$-matrix

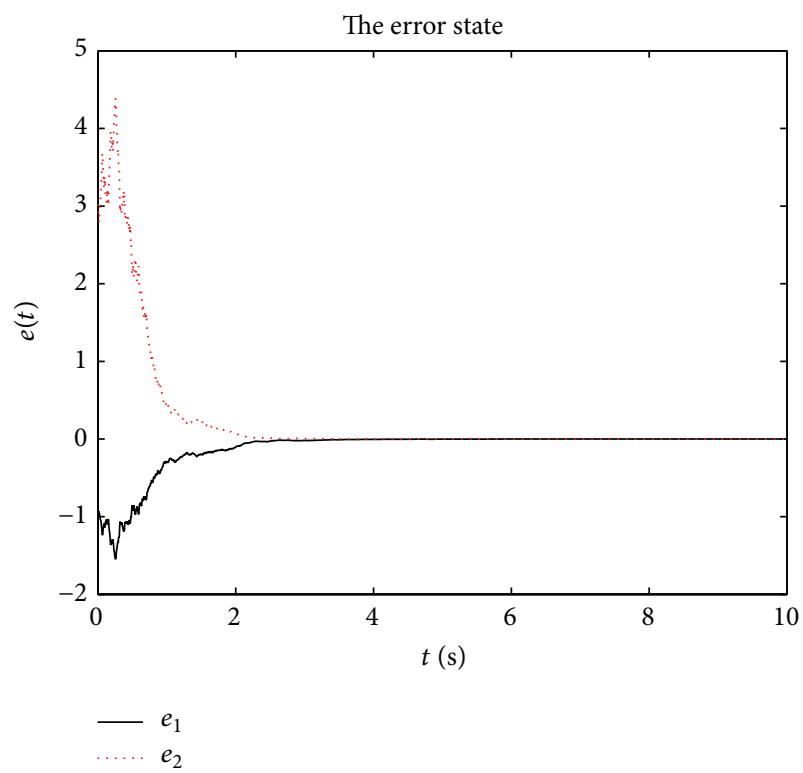

FIGURE 3: State trajectory of the error system.

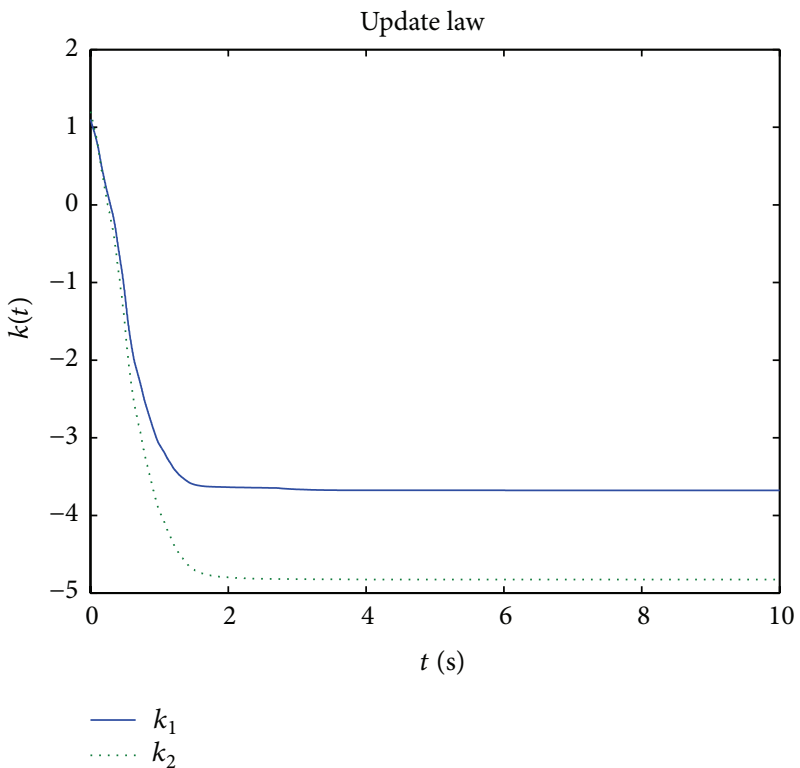

FIgURE 4: Update law.

approach and the stochastic analysis method, some synchronization criteria are obtained to ensure the stochastic synchronization for the neutral-type neural networks with multidelays. A numerical example is provided to illustrate the effectiveness of the result obtained in this paper.

\section{Conflict of Interests}

The authors declare that there is no conflict of interests regarding the publication of this paper. 


\section{Acknowledgments}

This work is partially supported by the Specialized Research Fund for the Doctoral Program of Higher Education under Grant no. 20120075120009 and the Natural Science Foundation of Shanghai under Grant no. 12ZR1440200.

\section{References}

[1] O. Arino and V. R. Nosov, "On stability of a class of neutral type functional differential equations," Mathematics and Computers in Simulation, vol. 45, no. 3-4, pp. 299-307, 1998.

[2] R. Rabah, G. M. Sklyar, and A. V. Rezounenko, "Stability analysis of neutral type systems in Hilbert space," Journal of Differential Equations, vol. 214, no. 2, pp. 391-428, 2005.

[3] W. Zhou, Y. Gao, D. Tong, C. Ji, and J. Fang, "Adaptive exponential synchronization in pth moment of neutral-type neural networks with time delays and Markovian switching," International Journal of Control, Automation and Systems, vol. 11, no. 4, pp. 845-851, 2013.

[4] Q. Zhu, W. Zhou, D. Tong, and J. Fang, "Adaptive synchronization for stochastic neural networks of neutral-type with mixed time-delays," Neurocomputing, vol. 99, pp. 477-485, 2013.

[5] R. Lu, H. Su, J. Chu, and A. Xue, "A simple approach to robust d-stability analysis for uncertain singular delay systems," Asian Journal of Control, vol. 11, no. 4, pp. 411-419, 2009.

[6] R. Lu, Y. Xu, and A. Xue, " $H_{\infty}$ filtering for singular systems with communication delays," Signal Processing, vol. 90, no. 4, pp. 1240-1248, 2010.

[7] Z. Wang, Y. Liu, L. Yu, and X. Liu, "Exponential stability of delayed recurrent neural networks with Markovian jumping parameters," Physics Letters, Section A: General, Atomic and Solid State Physics, vol. 356, no. 4-5, pp. 346-352, 2006.

[8] H. Huang, D. W. C. Ho, and Y. Qu, "Robust stability of stochastic delayed additive neural networks with Markovian switching," Neural Networks, vol. 20, no. 7, pp. 799-809, 2007.

[9] J. H. Park, O. M. Kwon, and S. M. Lee, "LMI optimization approach on stability for delayed neural networks of neutraltype," Applied Mathematics and Computation, vol. 196, no. 1, pp. 236-244, 2008.

[10] W. Zhou, H. Lu, and C. Duan, "Exponential stability of hybrid stochastic neural networks with mixed time delays and nonlinearity," Neurocomputing, vol. 72, no. 13-15, pp. 3357-3365, 2009.

[11] Y. Tang and J. Fang, "Adaptive synchronization in an array of chaotic neural networks with mixed delays and jumping stochastically hybrid coupling," Communications in Nonlinear Science and Numerical Simulation, vol. 14, pp. 3615-3628, 2009.

[12] M. Mariton and P. Bertrand, "Output feedback for a class of linear systems with stochastic jump parameters," IEEE Transactions on Automatic Control, vol. 30, no. 9, pp. 898-900, 1985.

[13] Y. Sun and J. Cao, "Stabilization of stochastic delayed neural networks with Markovian switching," Asian Journal of Control, vol. 10, no. 3, pp. 327-340, 2008.

[14] R. Lu, H. Li, and Y. Zhu, "Quantized $H_{\infty}$ filtering for singular time-varying delay systems with unreliable communication channel," Circuits, Systems, and Signal Processing, vol. 31, no. 2, pp. 521-538, 2012.

[15] D. Tong, Q. Zhu, W. Zhou, Y. Xu, and J. Fang, "Adaptive synchronization for stochastic T-S fuzzy neural networks with timedelay and Markovian jumping parameters," Neurocomputing, vol. 117, no. 6, pp. 91-97, 2013.
[16] Z. Wu, H. Su, J. Chu, and W. Zhou, "Improved result on stability analysis of discrete stochastic neural networks with time delay," Physics Letters, Section A: General, Atomic and Solid State Physics, vol. 373, no. 17, pp. 1546-1552, 2009.

[17] W. Zhou, Q. Zhu, P. Shi, H. Su, J. Fang, and L. Zhou, "Adaptive synchronization for neutral-type neural networks with stochastic perturbation and markovian switching parameters," IEEE Transactions on Cybernetics, vol. 44, no. 12, pp. 2848-2860, 2014.

[18] Z.-G. Wu, P. Shi, H. Su, and J. Chu, "Exponential synchronization of neural networks with discrete and distributed delays under time-varying sampling," IEEE Transactions on Neural Networks and Learning Systems, vol. 23, no. 9, pp. 1368-1376, 2012.

[19] Z.-G. Wu, P. Shi, H. Su, and J. Chu, "Delay-dependent stability analysis for switched neural networks with time-varying delay," IEEE Transactions on Systems, Man, and Cybernetics, Part B: Cybernetics, vol. 41, no. 6, pp. 1522-1530, 2011.

[20] Z.-G. Wu, P. Shi, H. Su, and J. Chu, "Stochastic synchronization of markovian jump neural networks with time-varying delay using sampled data," IEEE Transactions on Cybernetics, vol. 43, no. 6, pp. 1796-1806, 2013.

[21] Y. Xu, H. Su, and Y.-J. Pan, "Output feedback stabilization for Markov-based nonuniformly sampled-data networked control systems," Systems \& Control Letters, vol. 62, no. 8, pp. 656-663, 2013.

[22] Y. Xu, H. Su, Y.-J. Pan, Z.-G. Wu, and W. Xu, "Stability analysis of networked control systems with round-robin scheduling and packet dropouts," Journal of the Franklin Institute, vol. 350, no. 8, pp. 2013-2027, 2013.

[23] X. Wang, J.-A. Fang, A. Dai, W. Cui, and G. He, "Mean square exponential synchronization for a class of Markovian switching complex networks under feedback control and Mmatrix approach," Neurocomputing, vol. 144, pp. 357-366, 2014.

[24] D. Tong, Q. Zhu, W. Zhou, Y. Xu, and J. Fang, "Adaptive synchronization for stochastic T-S fuzzy neural networks with timedelay and Markovian jumping parameters," Neurocomputing, vol. 117, pp. 91-97, 2013.

[25] Z. Wang, H. Shu, J. Fang, and X. Liu, "Robust stability for stochastic Hopfield neural networks with time delays," Nonlinear Analysis: Real World Applications, vol. 7, no. 5, pp. 1119-1128, 2006.

[26] K. Gu, "An integral inequality in the stability problem of timedelay systems," in Proceedings of the 39th IEEE Conference on Decision and Control, pp. 2805-2810, Sydney, Australia, 2000.

[27] X. Mao and C. Yuan, Stochastic Differential Equations with Markovian Switching, Imperial College Press, London, UK, 2006.

[28] X. Wang, J.-A. Fang, A. Dai, and W. Zhou, "Global synchronization for a class of Markovian switching complex networks with mixed time-varying delays in the delay-partition approach," Advances in Difference Equations, vol. 2014, article 248, 2014. 


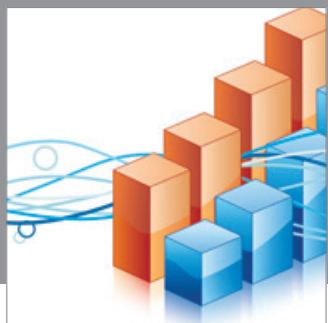

Advances in

Operations Research

mansans

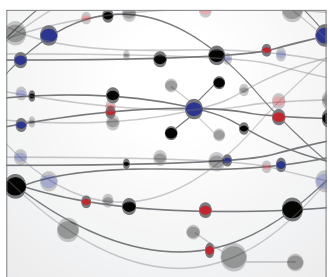

The Scientific World Journal
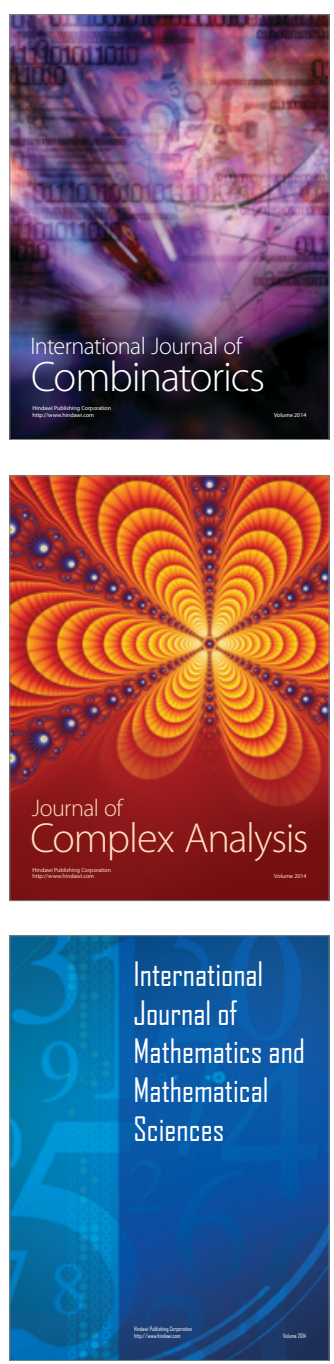
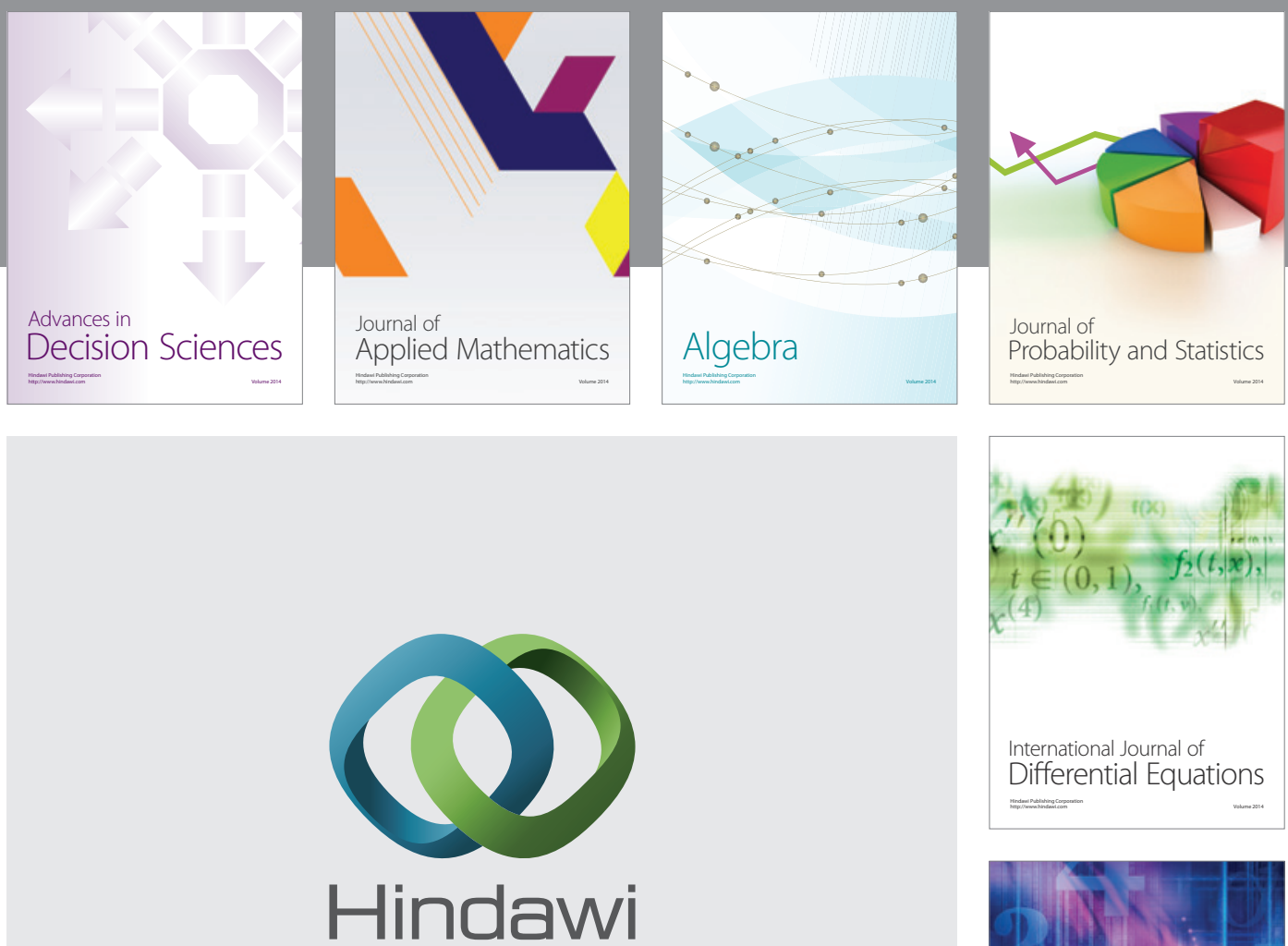

Submit your manuscripts at http://www.hindawi.com
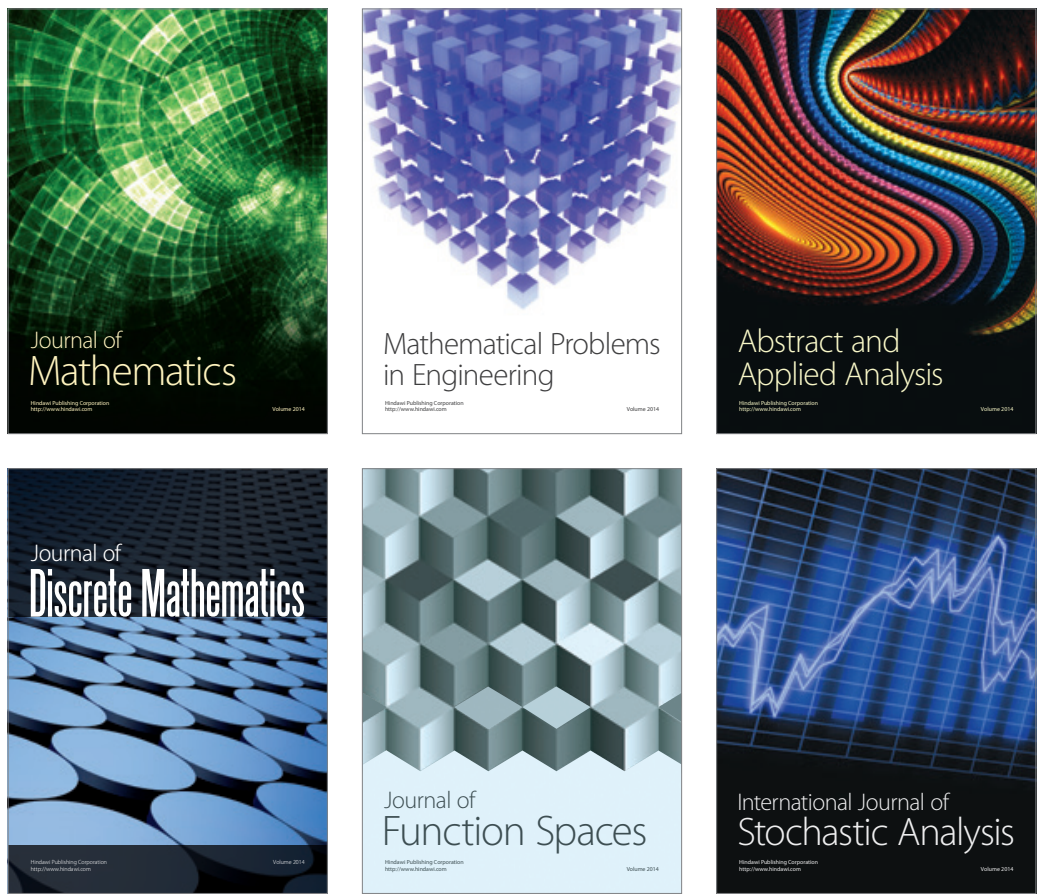

Journal of

Function Spaces

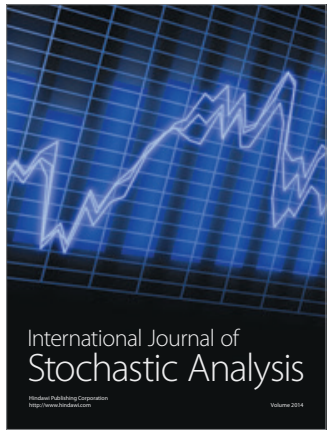

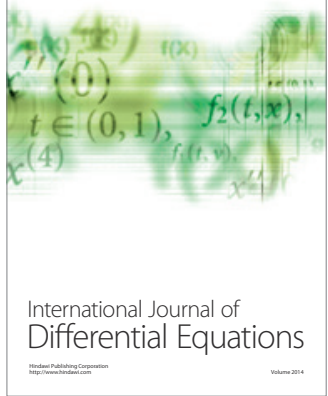
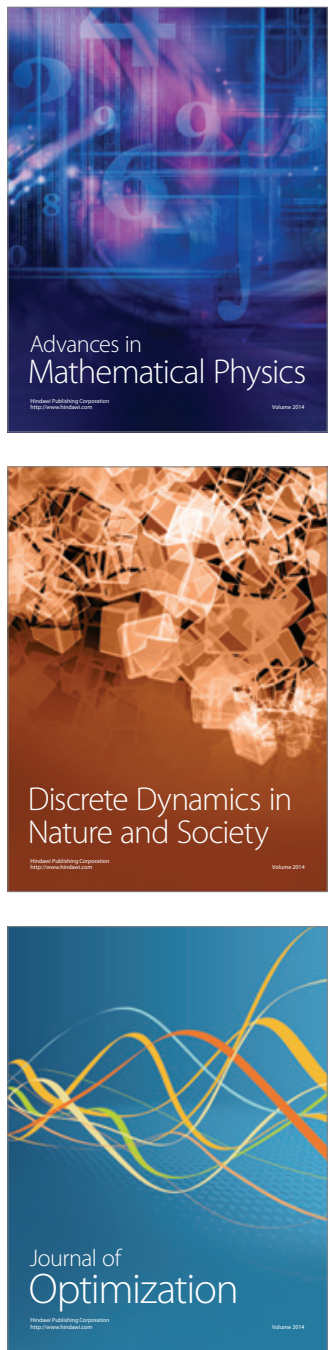